\title{
Anterior Tibial Tendon Transfer for Treatment of Recurrent Congenital Clubfoot Initially Treated According to Ponseti Method. Update and Systematic Review Of Literature
}

\author{
V. De Luna, A. Caterini, G. Gorgolini, M. Marsiolo, F. De Maio, P. Farsetti \\ Division of Orthopaedic Surgery, Department of Clinical Sciences and Translational Medicine, \\ University Tor Vergata, Rome, Italy
}

\section{CORRESPONDING AUTHOR: \\ Pasquale Farsetti \\ Department of Orthopaedic Surgery \\ University of Rome Tor Vergata \\ viale Oxford 81 \\ 00133 Rome, Italy \\ E-mail: farsetti@uniroma2.it}

DOI:

10.32098/mltj.02.2021.10

LEVEL OF EVIDENCE: 5

\begin{abstract}
SUMMARY
Background. Congenital clubfoot has a tendency to recur regardless the type of treatment; recurrences generally occur after a complete correction of the deformity. Anterior tibial tendon transfer (TATT) is commonly used for treatment of recurrent clubfeet in deformities initially treated according to Ponseti method, since extensive surgery has a high rate of poor results. The aim of our study was to analyze a series of papers, recently published, to evaluate the effectiveness of TATT for treatment of recurrent congenital clubfoot initially treated according to Ponseti method.

Methods. A literature search on the topic was performed by searching the databases Medline (Pubmed) and Cochrane Library, to select only articles from the recent literature (January 2000 to January 2021). The articles were screened for the presence of the following inclusion criteria: patients affected by recurrent congenital clubfoot originally managed by Ponseti method and treated by anterior tibial tendon transfer. All the patients affected by non-idiopathic congenital clubfoot and by residual deformities or neglected deformities were excluded.

Results. The initial search produced 123 studies from Medline database and 22 studies from Cochrane library database. After detailed evaluation based on inclusion and exclusion criteria, articles were screened and only 11 studies fulfilled the eligibility criteria of our study. All the selected articles were published from 2006 to 2020 and they included overall 331 patients (481 clubfeet).

Conclusions. We believe that TATT is an effective surgical procedure to treat recurrent clubfeet initially treated by Ponseti method, independent of the type of surgical technique. A second recurrence is absolutely not common, however it is more frequent when TATT is performed in younger patients. TATT seems to be effective also in late relapse, although in some cases an associated surgical procedure is necessary.
\end{abstract}

\section{KEY WORDS}

Anterior tibial tendon transfer; TATT; recurrent congenital clubfoot; relapse; tibialis anterior.

\section{INTRODUCTION}

The Ponseti conservative method for treatment of idiopathic clubfoot deformity is actually adopted all over the world. This method allows to obtain excellent results, although, as stated by Ponseti, the clubfoot has a stubborn tendency to relapse regardless the mode of treatment and "its wrongly assumed that relapses occur because the deformity has not been completely corrected" (1). The reliability of this conservative method has been proved by several clinical and radiological studies (2-6). The incidence of recurrence after a complete initial correction of the deformity using Ponseti method ranges from $7 \%$ to $56 \%$ (7). In a recent survey of the POSNA member, the incidence of clubfoot relapses managed by Ponseti method, was $<10 \%$ by $22 \%$ of the respondents, between $10 \%$ to $20 \%$ by $52 \%$ and between $20 \%$ to $40 \%$ by $25 \%$ (8). Anterior tibial tendon transfer 
(TATT) is commonly used for treatment of recurrent clubfeet, since extensive surgery has a high rate of poor results (9-12). Garceau (13) first described the surgical technique of TATT to the lateral side of the foot to correct relapsing clubfoot deformities. The author proposed to perform the TATT to the fifth metatarsal bone or the cuboid by three different incisions, pulling out the tendon from the extensor retinaculum at the ankle. Twenty years later, Ponseti and Smoley (14) modified the original technique, proposing to transfer the tendon on the third cuneiform, by only two incision leaving the tendon under the retinaculum. Hoffer et al., in two subsequent studies $(15,16)$ further modified the technique transferring the lateral half of the anterior tibial tendon by splitting it, to the cuboid to correct equinovarus deformity in cerebral palsied patients. These three different surgical techniques are tested in a cadaveric foot model in which the authors concluded that all three techniques may be useful and deliver varying degrees of increased forefoot pronation (17). However, currently the most common TATT procedure provides to reinsert the tendon to the third cuneiform, through a hole drilled in the ossified bone using two Kite's needles. The transferred tendon was prepared with a Bunnell-type suture and anchored to the plantar sole with a button (figure 1 A-E). A recent cadaver study suggests passing the sutures with a blunt needle to prevent damage to nerves and vessels of the plantar side of the foot (18). Ponseti and Smoley technique is performed making only two limited incisions on the dorsum of the foot
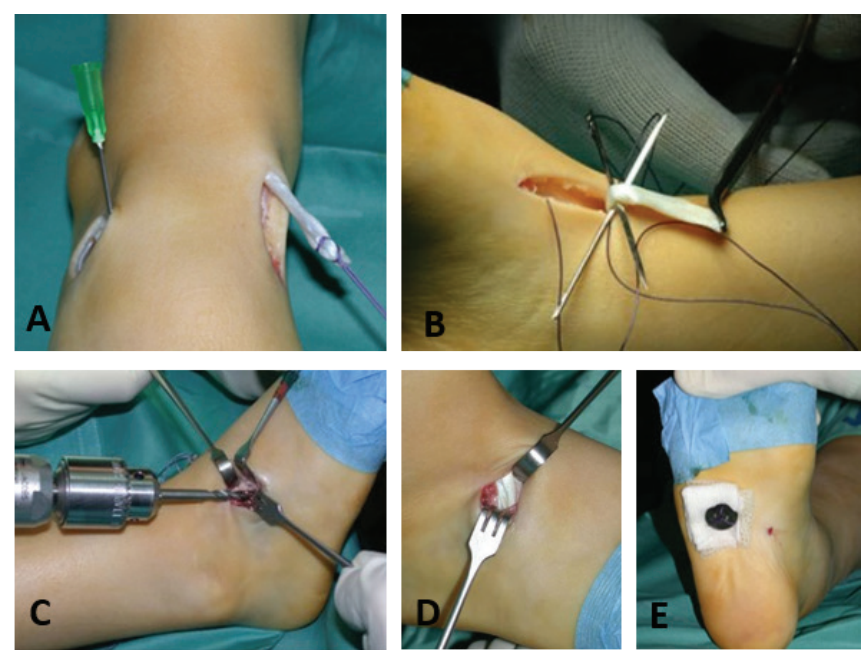

Figure 1. Ponseti and Smoley surgical technique. (A) Two small incisions are performed on the dorsum of the foot. (B) The tibialis anterior tendon is prepared with a Bunnel type suture. (C) A tunnel is drilled in the third cuneiform, (D) then the tendon is passed through the bone using two Kite's needles and (E) anchored to the plantar sole with a button. instead of the three more invasive incisions proposed by the original Garceau technique, avoiding to pull out the tendon from the extensor retinaculum. Regarding the anchor of the transferred tendon to the bone, some authors suggested different technique using bone anchors instead bone tunnel or bioabsorbable screw $(19,20)$.

Several retrospective studies reported that TATT seems to be the best EBM surgical procedure for treatment of recurrent clubfeet originally managed by Ponseti method; however, to the best of our knowledge, the majority of reported articles had level of evidence of III or IV. Only three studies were categorized as level II. Equally satisfactory results are observed by other authors that using TATT in recurrent clubfeet in patients initially treated by extensive posteromedial release or in rigid residual deformities $(11,12,21-26)$.

The aim of our study was to analyze a series of papers published from 2000 to present to evaluate the effectiveness of the anterior tibial tendon transfer for treatment of recurrent congenital clubfoot initially treated according to Ponseti method.

\section{METHODS}

To guide the review the Preferred Reporting Items for Systematic Reviews and Meta-Analyses (PRIS-MA) guidelines were used and adhered to $(27,28)$.

Studies comparing non-operative management to surgical manage-ment of recurrent clubfeet originally managed by Ponseti method were evaluated against the set inclusion criteria. To formulate the inclusion and exclusion criteria, the PICOT method was used (29) (table I).

\section{Search strategy and sources of information}

Authors of this review (VDL, AC, GG, MM, FDM, PF) performed a literature search about the topic by querying online databases. Studies were located by searching the databases Medline (Pubmed) and Cochrane Library. The search strategy covers PICO and was performed independently by each author on January 2021. Keywords and $\mathrm{MeSH}$ Terms were identified by a preliminary search and selected by discussion. The search was conducted using the following keywords assembled in various combination to obtain most pertinent articles: clubfoot, club foot, club-foot, clubfeet, club feet, club-feet, congenital clubfoot, talipes equinovarus, pes equinovarus, equinovarus, recurrent, relapse, relapsed, relapsing, residual, tendon transfer, anterior tibial tendon transfer, tibialis anterior tendon transfer, anterior tibial tendon, tibialis anterior, tatt, attt, att, dynamic supination. The following search queries were used: 1) (“2000/01/01”[Date - Publication]: “3000”[Date 
Table I. Inclusion and exclusion criteria (PICOT).

\begin{tabular}{lll}
\hline Population & Inclusion criteria & Exclusion criteria \\
\hline Intervention & $\begin{array}{l}\text { Patients affected by recurrent congenital clubfoot originally } \\
\text { managed by Ponseti method. }\end{array}$ & $\begin{array}{l}\text { Patients affected by non-idiopathic } \\
\text { congenital clubfoot. } \\
\text { Patients affected by residual deformities or } \\
\text { neglected congenital clubfoot. }\end{array}$ \\
\hline Comparison group & TATT in patients originally managed by Ponseti method. & Other surgical techniques. \\
& TATT in patients originally treated by surgical methods. & Not applicable. \\
\hline Outcome & surgical methods. & \\
\hline Time & Studies reporting clinical, radiographic and pedobarographic & Not applicable. \\
evaluation (Laaveg and Ponseti score; Dimeglio score; & \\
\hline AOFAS score). & Studies published prior to 2000. \\
\hline Language & Studies published from 2000 to 2020. & Letters. \\
\hline & Clinical Trials. & Case reports. \\
& Cohort Studies. & \\
\hline
\end{tabular}

- Publication]) anterior tibial tendon transfer for treatment of recurrent congenital clubfoot; 2) (“2000/01/01”[Date Publication]: "3000"[Date - Publication]) AND ("Clubfoot"[Mesh] OR "club feet"[Title/Abstract] OR "club foot"[Title/Abstract] OR "clubfoot"[Title/Abstract] OR "talipes equinovarus"[Title/Abstract] OR "Club-foot"[Title/Abstract] OR “pes equinovarus"[Title/Abstract]) AND ("recurrent"[Title/Abstract] OR "relapsing"[Title/Abstract] OR "relaps*"[Title/Abstract] OR "residual"[Title/Abstract]) AND ("Tendon Transfer"[Mesh] OR "anterior tibial tendon transfer"[Title/Abstract] "tibialis anterior tendon transfer" [Title/Abstract] OR "TATT" [Title/Abstract] OR “Tibialis Anterior"[Title/Abstract]); 3) Anterior tibial tendon; Clubfoot; Dynamic supination; Relapse; Tendon transfer; 4) (“2000/01/01"[Date - Publication]: "3000" [Date - Publication]) AND tibialis anterior tendon transfer AND clubfoot[TITLE/ABSTRACT] AND "relaps*"[TITLE/ABSTRACT]; 5) ("2000/01/01"[Date - Publication]: “3000"[Date - Publication]) AND anterior tibialis tendon transfer AND clubfoot[TITLE/ ABSTRACT] AND “relaps*"[TITLE/ABSTRACT]; To provide an update about the main topic, a publication date filter was applied to select only articles and review articles from the recent literature ranging from January 2000 to January 2021. Language restriction were applied to identify only English articles. No other filters were applied. In addition, a manual search was performed of the references cited in studies, reviews, and pediatric foot and ankle reference textbooks and university libraries.
The reviewers (VDL, AC, GG, MM, FDM, PF) retrieved the data and independently analyzed each selected study; instances of disagreement were resolved by the senior investigator (PF).

The articles were screened for the presence of the following inclusion criteria:

1. patients affected by recurrent congenital clubfoot originally managed by Ponseti method;

2. anterior tibial tendon transfer surgical technique;

3. studies providing an adequate level of evidence, including retrospective studies;

4. availability of full text.

The researches were excluded if providing information regarding:

1. studies on different technique than anterior tibial tendon transfer;

2. studies on patients affected by non-idiopathic congenital clubfoot;

3. studies on patients affected by residual deformities or neglected congenital clubfoot.

Figure 2 shows the PRISMA flowchart for study selection.

\section{RESULTS}

The initial search produced 123 studies from Medline database and 22 studies from Cochrane library database. One more article was included by the search after that the references were screened. Duplicated were filtered out and a total of 84 unique studies were obtained (68 from Medline 
Figure 2. Flow diagram of search process.

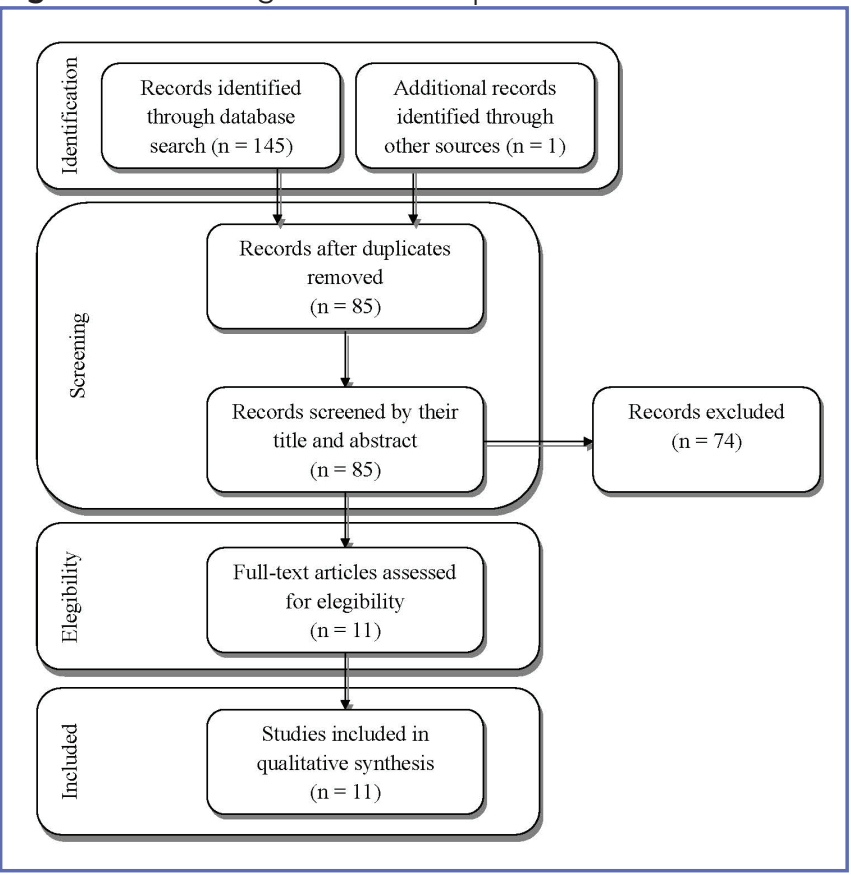

database, 16 from Cochrane library and 1 from references). After detailed evaluation based on inclusion and exclusion criteria, articles were screened and only 11 studies fulfilled the eligibility criteria of our study (30-40). The other studies were excluded for the following reasons: 70 articles did not meet the study design because the topic was not pertinent or have insufficient data or the deformities were initially treated by surgical procedures while 6 articles were published in a different language than in English. In conclusion, a total of 11 articles were enrolled in the present review (30-40). All the selected articles were published from 2006 to 2020 and they included overall 331 patients (481 clubfeet). Table II presents the list of reference of the studies, level of evidence, number of patients and clubfeet, age at surgery, surgical technique performed, combined TAL, age at follow-up, results analysis, possible second recurrence after TATT and conclusions.

\section{DISCUSSION}

Recurrent congenital clubfoot is caused by the same pathology that initiated the deformity. Stiff clubfeet with a severe atrophy of the leg muscles have a greater tendency to recur, in comparison to more flexible deformities $(1,41-45)$. The incidence of recurrence of congenital clubfoot is considerably decreased in the last 20 years for two main reasons, the greater widespread of the Ponseti method instead of surgical procedures and the better parent's compliance with bracing after casting. However, recurrence is still observed independently of the method of treatment performed, and usually occurs between 2 and 5 years of age; it generally rare after 5 years of age and extremely rare after 7 years of age (1). Few long term follow-up studies have been reported on the effectiveness of the anterior tibial tendon transfer to the lateral side of the foot to correct relapsed or relapsing clubfeet, initially treated according to Ponseti method based on a serial of casting performed following Ponseti technique and possible Achilles tendon tenotomy. In all these studies, the surgical technique of Ponseti and Smoley had been used. Farsetti et al. (30) reported satisfactory results in a series of 12 patients ( 16 clubfeet) surgically treated by TATT at an average age of 3.9 years. The results were clinically evaluated, according to the Laaveg and Ponseti point system based on pain, function, satisfaction, ROM, forefoot alignment and ability to walk. All the patients were also evaluated by radiographs and CT scan examinations. The authors concluded that TATT corrects and stabilizes relapsing clubfeet by restoring their normal function of foot dorsiflexion/eversion. Radiographic examinations and CT scan showed some anatomic anomalies in the treated clubfeet, in fact the cuneiforms and the cuboid were shifted more laterally than normal in spite of a persistent subluxation of the navicular bone. The authors pointed out the importance of the flexibility of the foot that represents the main condition for a successful final result, since this surgical procedure is based on the dynamic muscle balance of the forefoot. Similar results have been reported more recently in another long term follow-up study (35), in which the authors analyzed 14 patients (25 clubfeet) from the clinical and radiographic point of views and using pedobarographic analysis (peak pressures, total force distribution) and surface electromyography. The authors concluded that TATT is very effective at preventing additional relapse without affecting longterm foot function. The radiographic changes commonly observed in the operated feet did not correlate with the long-term functional outcomes. The third long-term followup study (32) reported the treatment results of late relapsing idiopathic clubfeet previously treated by the Ponseti method. The authors divided their 39 patients (60 feet) in five groups with an average age that ranged from 6 to 8.3 years; 56 clubfeet were surgically treated by TATT, in some cases associated to other surgical procedure as plantar fasciotomy, extensor hallicus longus recession or limited posterior release. They reported that only 5 cases needed a revision surgery and two of them a triple arthrodesis. The authors concluded that TATT is effective in late relapsed deformities, in some cases combined to other surgical procedures. 


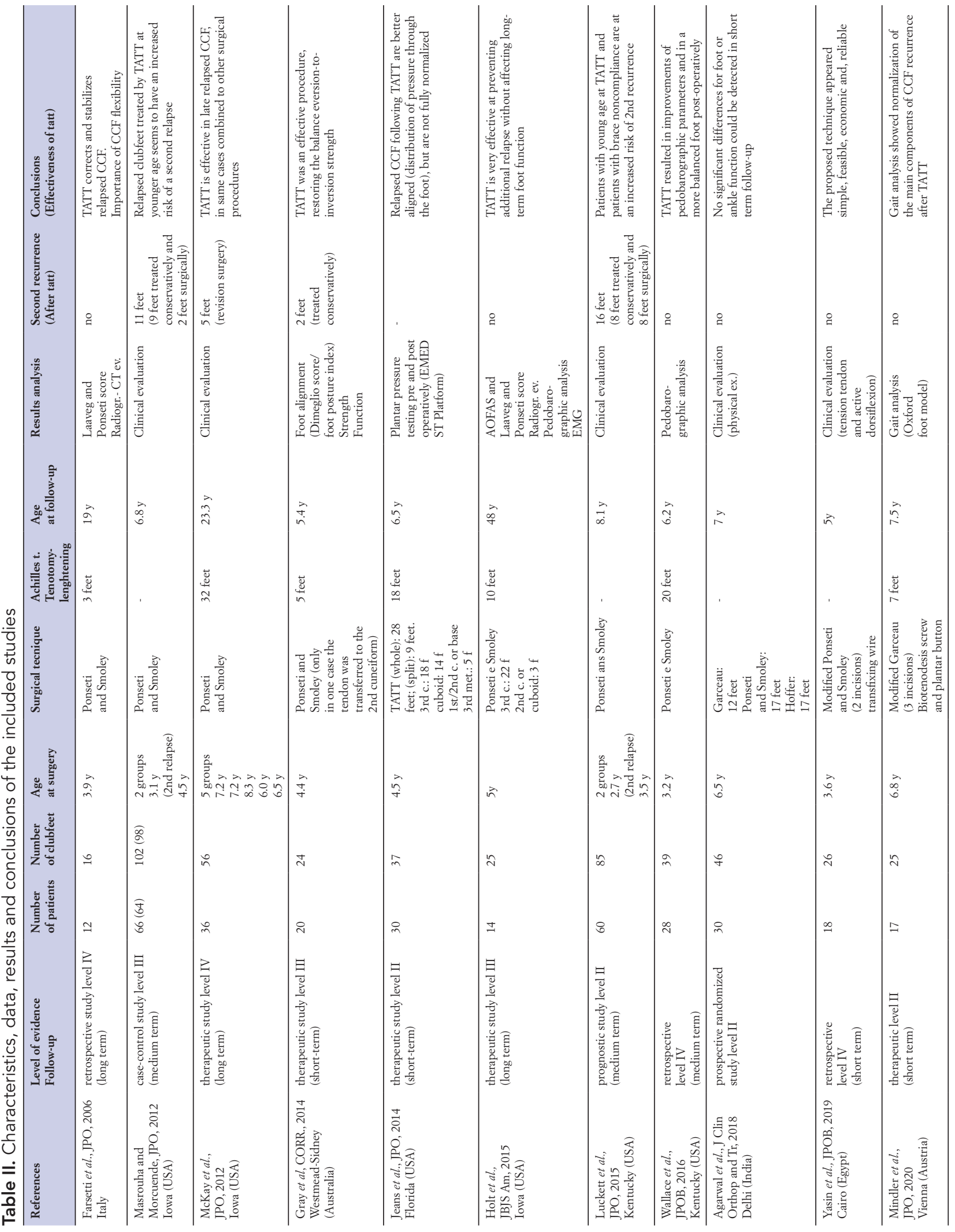


Despite $55 \%$ having residual deformity, at final follow-up, $80 \%$ of patients were without functional limitation.

Three further studies reported the medium-term followup results in as many series of patients affected by relapsed clubfeet treated by TATT, according to Ponseti and Smoley technique $(31,36,37)$. In two of them, the authors identified the deformities who presented further relapses after TATT procedure $(31,37)$. In both these articles, in which a considerable number of clubfeet were analyzed $(98+85$ feet), the authors agreed that clubfeet treated by TATT at younger age, showed an increased risk of a second relapse. However, the majority of the second relapses observed were treated conservatively. In the third study of this second group (medium-term follow-up study), the authors reported a series of 39 recurrent clubfeet treated by TATT and evaluated through a pedobarographic analysis and concluded that TATT resulted in improvements of pedobarographic parameters and in a more balanced foot postoperatively. The remaining articles are short term follow-up studies (33, $34,38-40$ ), that analyzed overall 115 patients (158 relapsed clubfeet) treated by TATT; contrary to previous studies, in these articles, the surgical techniques used were different (Ponseti and Smoley, Garceau and Hoffer) and some surgical technique variations have been proposed. Also these studies emphasized that TATT procedure is effective, restoring the balance eversion/inversion strength of the foot with an improvement of the distribution of the plantar pressure of the foot (34) and, at the gait analysis, a normalization of the main components of dynamic clubfoot recurrence was observed (39). Among them, Argawal et al. (40) conducted a prospective randomized study on three groups of patients with relapsed clubfoot (30 patients; 46 clubfeet), treated by TATT performed by Ponseti and Smoley technique in 17 feet, by Garceau technique in 12 feet and by Hoffer technique in another 17 feet. The average patient's age at surgery was 6.48 years and the average follow-up 5.49 months. The authors concluded that no significant differences for foot or ankle function could be detected using the three different surgical technique in short-term follow-up. Other two studies proposed a variation technique of the Ponseti and Smoley and Garceau technique respectively, reporting satisfactory results; Yasin et al. (38) performed two incisions as in the Ponseti and Smoley tech-

\section{REFERENCES}

1. Ponseti. Congenital clubfoot: Fundamentals of treatment (4 ed.) Oxford University Press, New York, 1996.

2. Ippolito E, Farsetti P, Caterini R, Tudisco C. Long-term comparative results in patients with congenital clubfoot nique, but modified the anchor of the transferred tibialis anterior tendon using a transfixing wire, while Mindler et al. (39), performed a three incisions as in the Garceau technique, but they fixed the tendon using both a Bio-Tenodesis screw and a plantar button.

Regarding the comparison group of studies including patients originally treated by surgical methods (11, 12, 21-26) instead of Ponseti method, the authors reported equally satisfactory results. However, they suggested to perform a recasting, according to Ponseti method, before TATT with the aim to soften the foot recurrent deformity that after surgery often appear to be stiff. Regarding the biases and limitations, this systematic review focused on 1 prospective randomized study, 1 prognostic study, 5 therapeutic study, 3 retrospective studies and 1 case-control study. Therefore, only few high quality evidence studies were included in this review. Literature search was performed with the aim to include all possible keywords to retrieve all published studies regarding our topic, however it is possible that some studies eligible for review were not identified due to publication bias. A language bias was also introduced as studies written in languages other than English were excluded.

In conclusion, from an accurate review of the literature, we believe that TATT is an effective surgical procedure to treat relapsed clubfeet. The majority of the authors prefer the Ponseti and Smoley technique, that has been used in all the studies with a long or medium follow-up, although the only paper that prospectively analyzes possible differences between the various techniques did not show any difference between them. A second recurrence is absolutely uncommon, however it is more frequent when TATT is performed in younger patients. TATT seems to be effective also in late relapse, although in some cases an associated surgical procedure is necessary. The pedobarographic analysis performed in some studies, showed an improvement of the plantar pressure after surgery as well as the only study on gait analysis performed with the Oxford foot model, showed a normalization of the main components of CCF recurrence after TATT.

\section{CONFLICT OF INTERESTS}

The authors declare that they have no conflict of interests.

treated with two different protocols. J Bone Joint Surg Am 2003;85:1286-94.

3. Ippolito E, Fraracci L, Caterini R, Di Mario M, Farsetti P. A radiographic comparative study of two series of skeletally 
mature clubfeet treated by two different protocols. Skeletal Radiol 2003;32:446-53.

4. Ippolito E, Fraracci L, Farsetti P, Di Mario M, Caterini R. The influence of treatment on the pathology of club foot. CT study at maturity. J Bone Joint Surg Br 2004;86:574-80.

5. Ippolito E, Fraracci L, Farsetti P, De Maio F. Validity of the anteroposterior talocalcaneal angle to assess congenital clubfoot correction. AJR Am J Roentgenol 2004;182:1279-82.

6. Farsetti P, De Maio F, Russolillo L, Ippolito E. CT study on the effect of different treatment protocols for clubfoot pathology. Clin Orthop Relat Res 2009;467:1243-9.

7. Holt JB, Westerlind B, Morcuende JA. Tibialis Anterior Tendon Transfer for Relapsing Idiopathic Clubfoot. JBJS Essent Surg Tech 2015;5:e16.

8. Hosseinzadeh P, Kiebzak GM, Dolan L, Zionts LE, Morcuende J. Management of Clubfoot Relapses With the Ponseti Method: Results of a Survey of the POSNA Members. J Pediatr Orthop 2019;39:38-41.

9. Ricciardi Pollini PT, Ippolito E, Tudisco C, Farsetti P. Congenital clubfoot: results of treatment of 54 cases. Foot and Ankle 1984;5:107-17.

10. Ippolito E, Ricciardi-Pollini PT, Tudisco C, Ronconi P. The treatment of relapsing clubfoot by tibialis anterior transfer underneath the extensor retinaculum. Ital J Orthop Traumatol 1985;11:171-7.

11. Garg S, Dobbs MB. Use of the Ponseti method for recurrent clubfoot following posteromedial release. Indian J Orthop 2008;42:68-72.

12. Al-Hilli AB. Ponseti method in the treatment of post-operative relapsed idiopathic clubfoot after posteromedial release. A short term functional study. Foot (Edinb) 2020;45:101721.

13. Garceau GJ. Anterior tibial tendon transposition in recurrent congenital clubfoot. J Bone Joint Surg Am 1940;22:932-36.

14. Ponseti IV, Smoley EN. Congenital Club Foot: the Results of Treatment. J Bone Joint Surg Am 1963;45:261-75.

15. Hoffer MM, Reiswig JA, Garrett AM, Perry J. The split anterior tibial tendon transfer in the treatment of spastic varus hindfoot of childhood. Orthop Clin North Am 1974;5:31-8.

16. Hoffer MM, Barakat G, Koffman M. 10-year follow-up of split anterior tibial tendon transfer in cerebral palsied patients with spastic equinovarus deformity. J Pediatr Orthop 1985;5:432-4.

17. Knutsen AR, Avoian T, Sangiorgio SN, Borkowski SL, Ebramzadeh E, Zionts LE. How do different anterior tibial tendon transfer techniques influence forefoot and hindfoot motion? Clin Orthop Relat Res 2015;473:1737-43.

18. Radler C, Gourdine-Shaw MC, Herzenberge JE. Nerve structures at risk in the plantar side of the foot during anterior tibial tendon transfer: a cadaver study. J Bone Joint Surg Am 2012;5(94):349-55.

19. Fennell CW, Ballard JM, Pflaster DS, Adkins RH. Comparative evaluation of bone suture anchor to bone tunnel fixation of tibialis anterior tendon in cadaveric cuboid bone: a biomechanical investigation. Foot Ankle Int 1995;16:641-5.

20. Wu KW, Huang SC, Kuo KN, Wang TM. The use of bioabsorbable screw in a split anterior tibial tendon transfer: a preliminary result. J Pediatr Orthop B 2009;18:69-72.

21. Nogueira MP, Ey Batlle AM, Alves CG. Is it possible to treat recurrent clubfoot with the Ponseti technique after postero- medial release?: a preliminary study. Clin Orthop Relat Res 2009;467:1298-305.

22. Dragoni M, Farsetti P, Vena G, Bellini D, Maglione P, Ippolito E. Ponseti Treatment of Rigid Residual Deformity in Congenital Clubfoot After Walking Age. J Bone Joint Surg Am 2016;98:1706-12.

23. Shah A, Mehta R, Aroojis A. The Ponseti method of clubfoot treatment in walking age children: is it effective? A study of 56 children from 1 to 10 years of age. J Pediatr Orthop B 2019;28:159-66.

24. Ezra E, Hayek S, Gilai AN, Khermosh O, Wientroub S. Tibialis anterior tendon transfer for residual dynamic supination deformity in treated club feet. J Pediatr Orthop B 2000;9:207-11.

25. Thompson GH, Hoyen HA, Barthel T. Tibialis anterior tendon transfer after clubfoot surgery. Clin Orthop Relat Res 2009;467:1306-13.

26. Lampasi M, Bettuzzi C, Palmonari M, Donzelli O. Transfer of the tendon of tibialis anterior in relapsed congenital clubfoot: long-term results in 38 feet. J Bone Joint Surg Br 2010;92:277-83.

27. PRISMA. PRISMA - Transparent Reporting of Systematic Reviews and Meta-analyses. PRISMA 2015.

28. Liberati A, Altman DG, Tetzlaff J, et al. The PRISMA statement for reporting systematic reviews and meta-analyses of studies that evaluate healthcare interven-tions: explanation and elaboration. BMJ 2009;339:b2700.

29. Riva JJ, Malik KM, Burnie SJ, Endicott AR, Busse JW. What is your research question? An introduction to the PICOT format for clinicians. J Can Chiropr Assoc 2012;56:167-71.

30. Farsetti P, Caterini R, Mancini F, Potenza V, Ippolito E. Anterior tibial tendon transfer in relapsing congenital clubfoot: long-term follow-up study of two series treated with a different protocol. J Pediatr Orthop 2006;26:83-90.

31. Masrouha KZ, Morcuende JA. Relapse after tibialis anterior tendon transfer in idiopathic clubfoot treated by the Ponseti method. J Pediatr Orthop 2012;32:81-4.

32. McKay SD, Dolan LA, Morcuende JA. Treatment results of late-relapsing idiopathic clubfoot previously treated with the Ponseti method. J Pediatr Orthop 2012;32:406-11.

33. Gray K, Burns J, Little D, Bellemore M, Gibbons P. Is tibialis anterior tendon transfer effective for recurrent clubfoot? Clin Orthop Relat Res 2014;472:750-8.

34. Jeans KA, Tulchin-Francis K, Crawford L, Karol LA. Plantar pressures following anterior tibialis tendon transfers in children with clubfoot. J Pediatr Orthop 2014;34:552-8.

35. Holt JB, Oji DE, Yack HJ, Morcuende JA. Long-term results of tibialis anterior tendon transfer for relapsed idiopathic clubfoot treated with the Ponseti method: a followup of thirty-seven to fifty-five years. J Bone Joint Surg Am 2015;97:47-55.

36. Wallace J, White H, Xi J, et al. Pedobarographic changes in Ponseti-treated clubfeet with and without anterior tibialis tendon transfer: changes due to growth and surgical intervention. J Pediatr Orthop B 2016;25:89-95.

37. Luckett MR, Hosseinzadeh P, Ashley PA, et al. Factors predictive of second recurrence in clubfeet treated by ponseti casting. J Pediatr Orthop 2015;35:303-6. 
38. Yasin ES, Amin HE, Ghani HA. A new anchoring technique for tibialis anterior tendon transfer. J Pediatr Orthop B 2020;29:379-81.

39. Mindler GT, Kranzl A, Radler C. Normalization of Forefoot Supination After Tibialis Anterior Tendon Transfer for Dynamic Clubfoot Recurrence. J Pediatr Orthop 2020;40:418-24.

40. Agarwal A, Jandial G, Gupta N. Comparison of three different methods of anterior tibial tendon transfer for relapsed clubfoot: A pilot study. J Clin Orthop Trauma 2020;11:240-4.

41. Ippolito E, Ponseti IV. Congenital club foot in the human fetus. A histological study. J Bone Joint Surg Am 1980;62:8-22.
42. Farsetti P, Dragoni M, Ippolito E. Tibiofibular torsion in congenital clubfoot. J Pediatr Orthop B 2012;21:47-51.

43. Ippolito E, Dragoni M, Antonicoli M, Farsetti P, Simonetti G, Masala S. An MRI volumetric study for leg muscles in congenital clubfoot. J Child Orthop 2012;6:433-8.

44. Ippolito E, De Maio F, Mancini F, Bellini D, Orefice A. Leg muscle atrophy in idiopathic congenital clubfoot: is it primitive or acquired? J Child Orthop 2009;3:171-8.

45. Dragoni M, Gabrielli A, Farsetti P, Bellini D, Maglione P, Ippolito E. Complex iatrogenic clubfoot: is it a real entity? J Pediatr Orthop B 2018;27:428-34. 